\title{
FLORISITIC COMPOSITION AND ECOLOGICAL CHARACTERISTICS OF FLORA OF TEHSIL SARAI NAURANG, DISTRICT LAKKI MARWAT, PAKISTAN
}

Fawad Khan ${ }^{1}$, Zahir Muhammad ${ }^{1}$, Tahseen Ullah ${ }^{1}$, Khushdil Khan ${ }^{2}$, Shabir Ahmad ${ }^{2}$, Asif Kamal $^{2}$ and Khafsa Malik ${ }^{2}$

\begin{abstract}
The current study was performed to determine the floristic composition and ecological characterization of the flora of tehsil Sarai Naurang. The floristic diversity of the plant consists of 143 species belonging to 47 families including 42 dicots and 5 monocots. Poaceae was the dominant family containing 18 species followed by Brassicaceae, Fabaceae, Asteraceae and Solanaceae. Therophytes were dominant class having 89 species (62.23\%) followed by Microphanerophytes with 19 species $(13.28 \%)$, Hemicryptophytes with 13 species $(9.09 \%)$, Chamaephytes 8 species $(5.59 \%)$, Geophytes 7 species $(4.89 \%)$, Nanophanerophytes with 6 species $(4.19 \%)$ and Megaphanerophytes with 1 species $(0.69 \%)$. Leaf size of most plant species was reduced indicating an adaptation to arid climatic conditions. Nanophylls had the largest leaves, followed by Microphylls (40 species, $27.97 \%$ ), Leptophylls (34 species, $23.77 \%$ ), Mesophylls (15 species, $10.48 \%$ ), Megaphylls (2 species, $1.39 \%$ ), and Macrophylls (1 species $0.69 \%$ ).
\end{abstract}

Keywords: Ecological characteristics, Floristic composition, Leaf size, Sarai Naurang, Vegetations.

Citation: Khan, F.; Z.Muhammad; T.Ullah; K.Khan; S.Ahmad; M.J.Khan; A.Kamal and K.Malik. 2021. Florisitic Composition and Ecological Characteristics of Flora of Tehsil Saari Naurang, District Lakki Marwat, Pakistan. Pak. J. Weed Sci. Res., 27 (3):263-275.

\footnotetext{
${ }^{1} 1$ Department of Botany university of Peshawar,Pakistan

2 Department of Plant Sciences, Quaid-i-Azam University Islamabad, Pakistan

3 The University of Agriculture Peshawar, Pakistan

4 Department of Environmental Science, Shaheed Benazir Bhutto University, Sharingal, Dir Upper

Corresponding author: Email address: fawadbotany@gmail.com
} 


\section{Introduction}

District Lakki Marwat is one of the southern district of Khyber Pakhtunkhwa, Pakistan, extending from $32^{\circ} 53^{\prime}$ North Latitudes and $70^{\circ}-23^{\circ}$ to $71^{\circ}-16^{\circ}$ East Longitudes. Location of Lakki Marwat is in such a way that in the north side there is a district Bannu, southeast the Marwat range separate Lakki Marwat from D.I Khan district, while in the east the Karak niazi range separates it from Mianwali district. It is surrounded by Waziristan on the west and southwest which separate it from district Tank and the tribal area adjoining south Waziristan agency and Tank district (Ahmad et al. 2021; Ahmad et al. 2019).

Sarai Naurang is one of the tehsil of district Lakki Marwat. It is located at northrern area of district Lakki Marwat. It is extending from $32^{\circ} 44^{\prime} 2$ North latitude $70^{\circ} 42^{\prime} 50$ East longitude. It is 284 meters above sea level. The dominant flora of study area is Acacia nilotica, Morus alba, Morus nigra and Dalbergia sisso. The flora of a region refers to the

\section{Materials and Methods}

The field data were collected during the period 2018-19 for establishing the species composition and the extent of plants in the tehsil Sari Naurang, Lakki Marwat. Collected plants were dried, preserved in newspapers, and identified with the help of flora of Pakistan (Nasir and Ali, 1971, 2007; Ali and Qaisar, 1995-2009) and by comparing with the already identified plant specimens at

\section{Result and Discussion}

\section{Floristic characteristics}

Results and discussions are mentioned here for the floristic composition of species. The floristic diversity of plants consists of 143 species belonging to 47 different families. Poaceae was found to be the most dominant family having 18 species, followed by Brassicaceae 11 species, Fabaceae 10 species, Asteraceae 9 species and Solanaceae 8 species while remaining Families had 1 collections of plants species in a particular region. It varies from other vegetations which relates to plant size, species' population distribution, and species importance (Samad et al. (2018), Ahmad et al. (2019) studied floristic, frequency and vegetationbiological spectra which providing information about most dominant life forms (Therophytes 31.25\%, and hemicryptophytes $28.13 \%$ ). The floristic survey gives the information about plants importance for people which are used as drugs, oil and construction works. The identification of plants is very important because it shows and helps in counting plants species and its location in an area (Ali et al. 2018; Ahmad et al. 2020). Floristic composition and ecological characteristic of various parts of Pakistan are explored by Fazal et al. (2010). Nasir et al. (2002), Shah et al. (2014). Sher and Khan (2007). and Khan et al. (2014). From all these surveys, it is clear that no floristic survey had carried in the tehsil Sai Naurang. Therefore the present survey was conducted to analyze and show the floristic composition and ecological characteristics of plants of this area.

herbarium, Department of Botany, University of Peshawar, Pakistan. Plants were mounted over standard herbarium sheets. Floristic composition and ecological compositions list for all species was prepared. Raunkiaer (1934) and Husain (1954) categorised plant species based on leaf size and life types (1989).

to 4 species in each (Fig:3, Table 1 and Table 2). The most common species are Alhagi maurorum, Avena fatua, Convolvulus arvensis, Euphorbia helioscopia, Medicogo sativa, Rununculus muricatus, Saueda fruiticosa, Brassica campestris, Morus alba, Morus nigra, and Eucalyptus camaldulensis etc. Most of the plants were annual. The primary and dominant category was dicots 42 
families, followed by monocots 5 families, according to a floral research. Manhas et al., (2010) and Wariss et al. (2014) reported dicots as the dominant group in their study area and found similar results. There were 103 herbivores, 20 shrubs, and 20 trees among the 143 species). Khan et al. (2017), Durrani et al. (2005), Marwat \&
Qureshi (2000), Musharaf et al. $(2014 ; 2011)$ and Parveen et al. (2008) also reported these plants in many othere regions of the country. The finding result are online and assisted by Pakistan Flora (Nasir and Ali, 19701989; Ali and Nasir 1989-1992; Ali and Qaiser, (1995-2015) which also shown by the plants genus to be dominant.

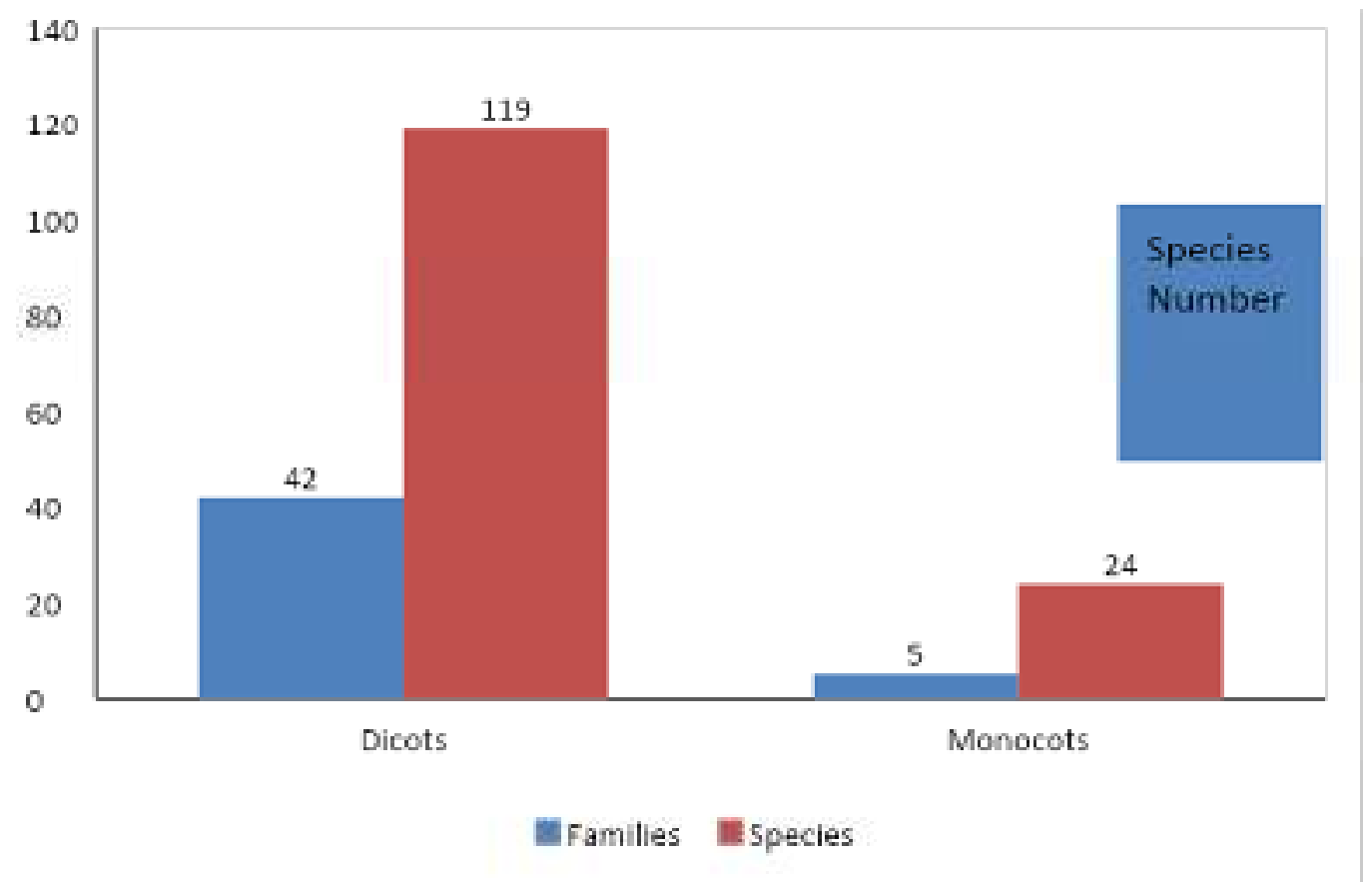

Fig. 2: Number of families and species of plant in tehsil Sari Naurang.

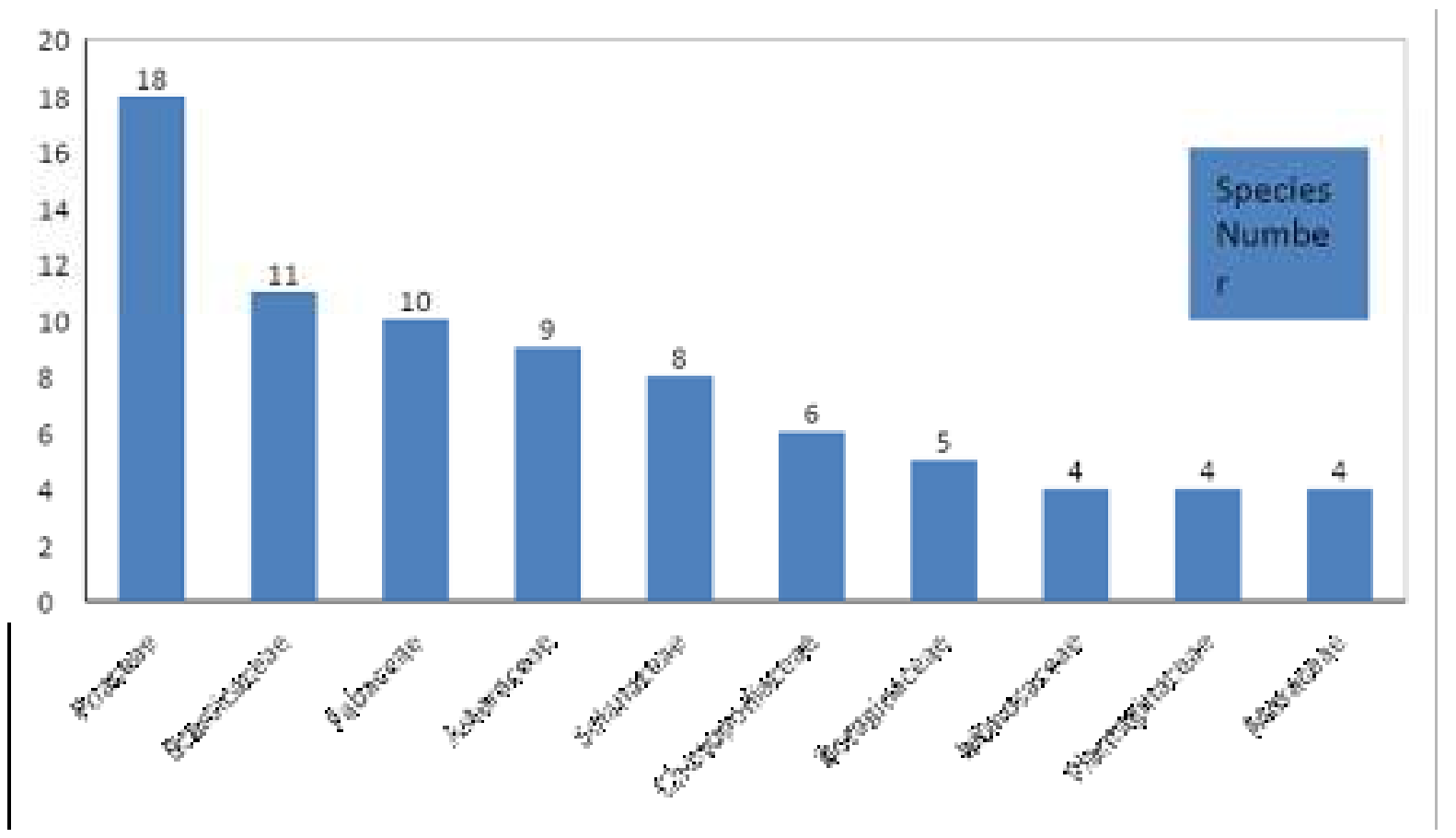

Fig. 3: Leading families showing number of species in tehsil Sari Naurang. 


\section{Plant habit}

Based on plant habit $103(72.02 \%)$ species were herbs, 20 species $(13.98 \%)$ shrubs and 20 species (13.98\%) were considered as trees (Fig: 4). Shuaib et al. (2014) and Qureshi et al. (2014) also reported herbs as the most dominant plant habit while studying spring flora of District Dir and Kanpur Dam, Respectively.
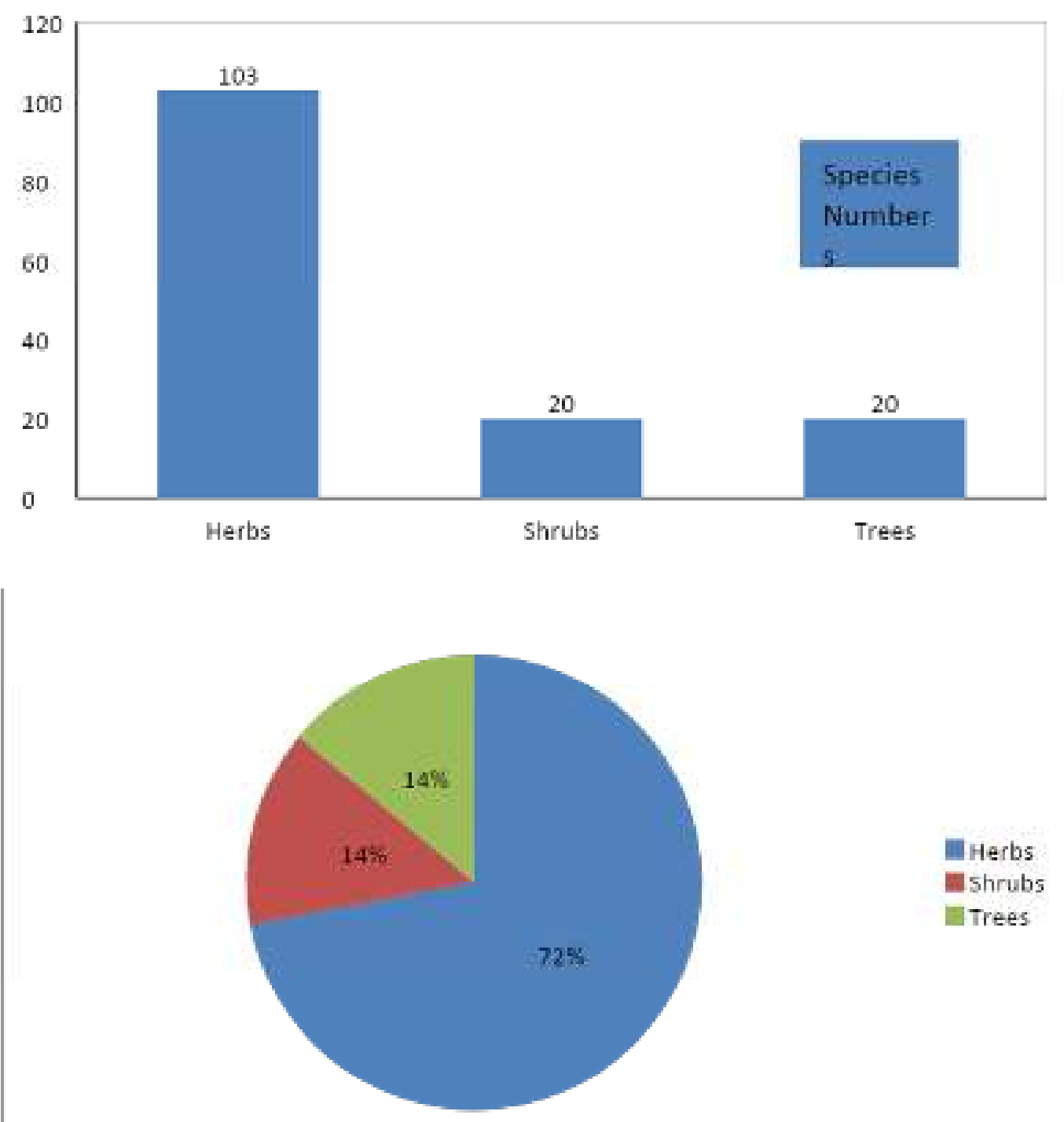

Fig.4: Bar and Pie diagram showing numbers and percentage of various plant habits.

\section{LIFE FORM SPECTRUM:}

The life form spectrum study declares the plants adaptability to climatic conditions. Accordingly to different ecosystem and various plants localities the life form of species is different with each other. Raunkiaer prepared life form classification based upon variation in plant structure during unfavorable climatic conditions. After studying
Raunkiaer classification, it was concluded that therophytes (89 spp., $62.23 \%$ ) was the most dominant life form in the district. After that microphanerophytes (19 spp., 13.28\%), hemicryptophytes (13 spp., 9.09\%) chamaephytes (8 Spp., 5.59\%) Geophytes (7 Spp., $4.89 \%$ ), nanophanerophytes (6 Spp., 4.19\%) and 
Megaphanerophytes (1 Sp., $0.69 \%$ ) (Fig: 5). The present study have good similarities with Ahmad et al. (2019). According to their floristic life form spectra, Therophytes were dominant in the area $(31.25 \%)$, followed by Hemicryptophytes (28.13\%). The dominancy of Therophytes also proved by Samadet al. (2018)30 species $(37.5 \%)$, Hussain and Chaudhri (2009)(55\%), Hussain et al. (2009) (53 spp, 85.48\%), Sher and Khan (2007) (86 spp. 38.56\%), Durraniet al (2005),

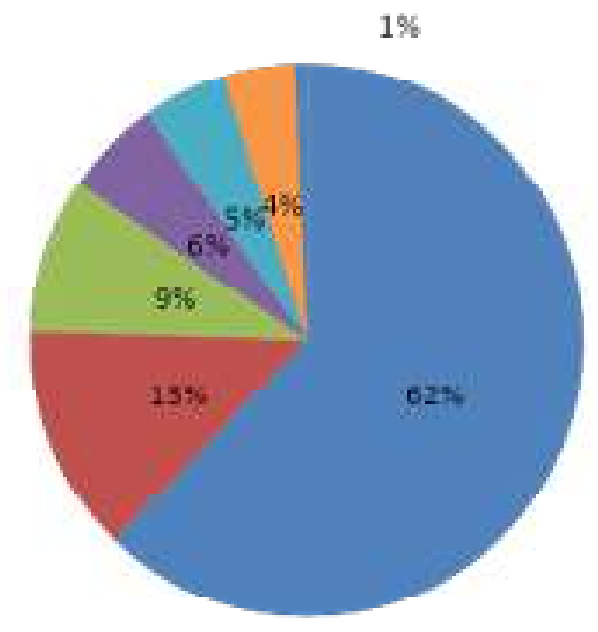

Nasir et al. (2002), Chain \& Castro (1959), Batalha \& Martins (2002), Badshah et al. (2013) and Musharaf et al. (2011). The dominancy of life form in study area shows adaptation to arid and harsh climatic condition which is capable to tolerate the unfavorable conditions. Some plants life form vary in different regions due to variations in environmental conditions. Therophytic plants completely shed even a little change in climatic condition.

\section{Fig. 5: Different life form classes showing percentage of species}

\section{LEAF SIZE SPECTRUM:}

Leaf size classes are very important for plants associations. Leaf size spectrum also helps in determination of plant habits and their relations with climate. The leaf spectra of Sari Naurang town reveled that nanophyll (50 Spp., $34.96 \%$ ) were the leading leaf size followed by Microphyll (40 Spp., 27.97\%), leptophyll (34 Spp., 23.77\%) mesophyll (15 spp., 10.48\%) megaphyll (2 spp., 1.39\%) and macrophyll (1 spp., $(0.69 \%)$ (Fig: 6). Our results showed clear resemblance with Badshahet al. (2013) who examined floral floristic composition, environmental variations and life form spectrum of rangeland district tank, Pakistan. Leaf spectra revealed that nanophylls were the most prevalent leaf size. Rather than Hussain et al. (2009), Hussain and Chaudhri (2009), Nasir and Sultan (2002). In any ecological area, leaf size is connected to environmental changes (Batalha and Martins, 2004). The size of leaf of an area reveals current climatic variations, however it cannot be used to shorten leaf 


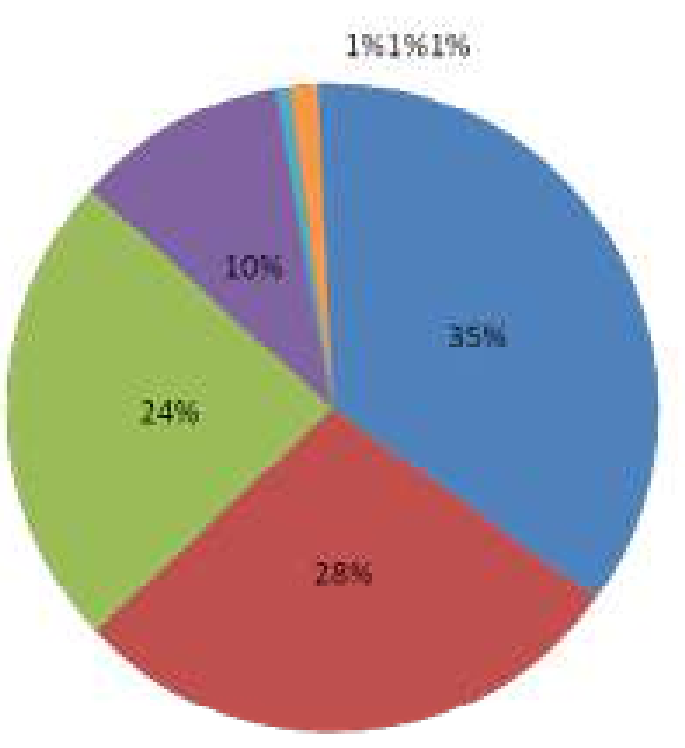

Fig.6: Different leaf size classes showing percentage of species.

Table 1. Summary of Characteristics of Flora of tehsil Sari Naurang

\begin{tabular}{|c|c|c|c|}
\hline S. No & Ecological Characteristics & Number & Percentage \\
\hline \multicolumn{4}{|c|}{ Flora } \\
\hline $\mathrm{I}$ & Total Families & 47 & 24.74 \\
\hline Ii & Total Species & 143 & 75.26 \\
\hline \multicolumn{4}{|c|}{ Habit } \\
\hline $\mathrm{I}$ & Herbs & 103 & 72.02 \\
\hline Ii & Shrubs & 20 & 13.98 \\
\hline iii & Trees & 20 & 13.98 \\
\hline \multicolumn{4}{|c|}{ Life form Spectra } \\
\hline i & Therophytes & 89 & 62.23 \\
\hline ii & Microphanerophytes & 19 & 13.28 \\
\hline Iii & Hemicryptophytes & 13 & 9.09 \\
\hline IV & Chamaephytes & 8 & 5.59 \\
\hline v & Geophytes & 7 & 4.89 \\
\hline $\mathrm{Vi}$ & Nanophanerophyte & 6 & 4.19 \\
\hline vii & Megaphanerophytes & 1 & 0.66 \\
\hline \multicolumn{4}{|c|}{ 4. $\quad$ Leaf size Spectra } \\
\hline $\mathrm{i}$ & Nanophyll & 50 & 34.96 \\
\hline Ii & Microphyll & 40 & 27.97 \\
\hline iii & Leptophyll & 34 & 23.77 \\
\hline
\end{tabular}




\begin{tabular}{|l|l|l|l|}
\hline iv & Mesophyll & 15 & 10.48 \\
\hline v & Macrophyll & 1 & 0.69 \\
\hline vi & Megaphyll & 2 & 1.39 \\
\hline vii & Aphyllous & 1 & 0.69 \\
\hline
\end{tabular}

Table -2. Floristic composition and ecological characteristics of flora of tehsil Sari Naurang, district Lakki Marwat

\begin{tabular}{|c|c|c|c|c|c|c|}
\hline So & Family & $\begin{array}{l}\text { Spp. } \\
\text { No }\end{array}$ & Species Name & Habit & $\begin{array}{l}\text { Leaf } \\
\text { Size }\end{array}$ & $\begin{array}{l}\text { Life } \\
\text { Form }\end{array}$ \\
\hline \multicolumn{7}{|c|}{ A. Monocots } \\
\hline \multirow[t]{2}{*}{1} & \multirow{2}{*}{ Alliaceae } & 1 & Allium cepa L. & Herb & $\mathrm{N}$ & G \\
\hline & & 2 & Allium griffithianum Boiss. & Herb & $\mathrm{N}$ & G \\
\hline 2 & $\begin{array}{l}\text { Arecacea } \\
\text { e }\end{array}$ & 3 & Nannorrhops ritchiana (Griff) & Shrub & Mac & $\mathrm{Mp}$ \\
\hline 3 & $\begin{array}{l}\text { Asphodel } \\
\text { aceae }\end{array}$ & 4 & Asphodelous tennifoliusL. & Herb & L & Th \\
\hline \multirow[t]{2}{*}{4} & \multirow{2}{*}{$\begin{array}{l}\text { Cyperac } \\
\text { eae }\end{array}$} & 5 & Cyperus rotundus $\mathrm{L}$. & Herb & $\mathrm{L}$ & MP \\
\hline & & 6 & $\begin{array}{l}\text { Fimbristylis dichotoma (L.) } \\
\text { Vahl }\end{array}$ & Herb & L & Th \\
\hline \multirow[t]{13}{*}{5} & \multirow[t]{13}{*}{ Poaceae } & 7 & Avena fatua $\mathrm{L}$. & Herb & $\mathrm{L}$ & Th \\
\hline & & 8 & Cynodon arcuatus]. Presl & Herb & $\mathrm{L}$ & $\mathrm{H}$ \\
\hline & & 9 & Cenchrus ciliaris L. & Herb & L & $\mathrm{Ch}$ \\
\hline & & 10 & Cynodon dactylon (L.) Pers & Herb & $\mathrm{L}$ & $\mathrm{H}$ \\
\hline & & 11 & $\begin{array}{l}\text { Cymbopogon jwarancusa } \\
\text { (Jones) } \\
\text { Schult. }\end{array}$ & Herb & Mic & Th \\
\hline & & 12 & $\begin{array}{l}\text { Dichanthium annulatum } \\
\text { (Frossk.) } \\
\text { Stapf. }\end{array}$ & Herb & Mic & $\mathrm{H}$ \\
\hline & & 13 & $\begin{array}{l}\text { Dectyloctenium aegyptium } \\
\text { (L.)Wild }\end{array}$ & Herb & $\mathrm{N}$ & Th \\
\hline & & 14 & Hordeun murinum L. & Herb & $\mathrm{N}$ & Th \\
\hline & & 15 & Hardium volgare L. & Herb & Mic & $\mathrm{H}$ \\
\hline & & 16 & Imperata cylindrical L. & Herb & $\mathrm{N}$ & $\mathrm{G}$ \\
\hline & & 17 & Oryza sativa L. & Herb & Mic & Th \\
\hline & & 18 & Poa annua L. & Herb & L & Th \\
\hline & & 19 & Polypogon monspeliensis L. & Herb & Mic & $\mathrm{H}$ \\
\hline
\end{tabular}


270 Fawad Khan, Zahir Muhammad et. al. Florisitic Composition and Ecological ...

\begin{tabular}{|c|c|c|c|c|c|c|}
\hline & & 20 & Phalaris minor (Retz). & Herb & Mic & $\mathrm{H}$ \\
\hline & & 21 & Paspalum paspalodes (L.) & Herb & Mic & Th \\
\hline & & 22 & Rostraria cristata (L.) Tzvelev & Herb & $\mathrm{N}$ & Th \\
\hline & & 23 & Triticum eastivum L. & Herb & Mic & Th \\
\hline & & 24 & Zea mays L. & Herb & Mes & Th \\
\hline \multicolumn{7}{|c|}{ B. Dicots } \\
\hline 6 & $\begin{array}{l}\text { Acanthac } \\
\text { eae }\end{array}$ & 25 & Justicia adhatoda L. & Shrub & $\mathrm{N}$ & $\mathrm{Mp}$ \\
\hline 7 & $\begin{array}{l}\text { Aizoacea } \\
\text { e }\end{array}$ & 26 & Trianthema protulacastrum L. & Herb & $\mathrm{N}$ & Th \\
\hline \multirow[t]{3}{*}{8} & \multirow{3}{*}{$\begin{array}{l}\text { Amaran } \\
\text { thacea }\end{array}$} & 27 & Aerva lanata L. & Herb & $\mathrm{N}$ & Th \\
\hline & & 28 & Amaranthus viridis (L.)Medik & Herb & $\mathrm{N}$ & Th \\
\hline & & 29 & Amaranthus graecizans L. & Herb & $\mathrm{L}$ & Th \\
\hline \multirow[t]{2}{*}{9} & \multirow{2}{*}{$\begin{array}{l}\text { Anacardi } \\
\text { acaeae }\end{array}$} & 30 & Mangifera indica L. & Tree & Mes & Th \\
\hline & & 31 & $\begin{array}{l}\text { Schinus } \quad \text { terebinthifolius } \\
\text { Raddi. }\end{array}$ & Tree & $\mathrm{N}$ & Th \\
\hline \multirow[t]{2}{*}{10} & \multirow[t]{2}{*}{ Apiaceae } & 32 & Torilis leptophylla (L.) Rchb.f. & Herb & $\mathrm{N}$ & Th \\
\hline & & 33 & Ammi visnaga (L). Lam. & Herb & $\mathrm{N}$ & Th \\
\hline \multirow[t]{2}{*}{11} & \multirow{2}{*}{$\begin{array}{l}\text { Apocyna } \\
\text { ceae }\end{array}$} & 34 & Periploca aphyla Dence. & Shrub & $A p$ & $\mathrm{~Np}$ \\
\hline & & 35 & Periploca calophylla (Wight) & Herb & Mes & Th \\
\hline \multirow[t]{2}{*}{12} & \multirow[t]{2}{*}{$\begin{array}{l}\text { Asclepia } \\
\text { daceae }\end{array}$} & 36 & $\begin{array}{l}\text { Calotropis procera (Willd) } \\
\text { R.Br. }\end{array}$ & Shrub & Mes & $\mathrm{Ch}$ \\
\hline & & 37 & Caralluma tuberculataN.E.Br. & Herb & $\mathrm{N}$ & $\mathrm{G}$ \\
\hline \multirow[t]{9}{*}{13} & \multirow{9}{*}{$\begin{array}{l}\text { Asterace } \\
\text { ae }\end{array}$} & 38 & Cirsium arvense(L.) Scop. & Herb & Mic & Th \\
\hline & & 39 & $\begin{array}{l}\text { Conyza canadensis } \quad \text { (L.) } \\
\text { Croquist. }\end{array}$ & Herb & Mic & Th \\
\hline & & 40 & Chrysanthemum indicum L. & Herb & $\mathrm{N}$ & $\mathrm{H}$ \\
\hline & & 41 & $\begin{array}{ll}\text { Centaurea } & \text { iberica } \\
\text { Trevir. \& Spreng. } & \end{array}$ & Herb & $\mathrm{N}$ & Th \\
\hline & & 42 & $\begin{array}{l}\text { Lactuca } \\
\text { (Boiss.) Boiss. }\end{array}$ & Herb & $\mathrm{N}$ & Th \\
\hline & & 43 & Launaea procumbens L. & Herb & Mes & Th \\
\hline & & 44 & Parthenium hysterophorus L. & Herb & Mes & Th \\
\hline & & 45 & Sonchus asper (L.) Hill & Herb & Mes & Th \\
\hline & & 46 & Tragopogon gracilis D.Done. & Herb & $\mathrm{N}$ & $\mathrm{H}$ \\
\hline \multirow[t]{4}{*}{14} & \multirow{4}{*}{$\begin{array}{l}\text { Boragen } \\
\text { aceae }\end{array}$} & 47 & Anchusa arvensis L. M.Bieb. & Herb & Mic & Th \\
\hline & & 48 & Cordia myxza (Engl.) & Herb & Mic & Th \\
\hline & & 49 & Heliotropium crispumL. & Herb & $\mathrm{N}$ & Th \\
\hline & & 50 & Heliotropium europium L. & Herb & Mic & Th \\
\hline
\end{tabular}




\begin{tabular}{|c|c|c|c|c|c|c|}
\hline & & 51 & Nonea edgworthii L. & Herb & $\mathrm{N}$ & $\mathrm{H}$ \\
\hline \multirow[t]{11}{*}{15} & \multirow{11}{*}{$\begin{array}{l}\text { Brassica } \\
\text { ceae }\end{array}$} & 52 & Brassica campestris L. & Herb & $\mathrm{N}$ & Th \\
\hline & & 53 & Brassica juncea (L.)Czern & Herb & $\mathrm{N}$ & Th \\
\hline & & 54 & Brassica oleracea L. & Herb & $\mathrm{N}$ & Th \\
\hline & & 55 & Brassica rapa L. & Shrub & L & Th \\
\hline & & 56 & Cardaria halepensisL. & Herb & $\mathrm{N}$ & Th \\
\hline & & 57 & Coronopus didymus (L.) Persl & Herb & $\mathrm{N}$ & Th \\
\hline & & 58 & Eruca sativa Mill. & Herb & Mic & NP \\
\hline & & 59 & Malcolmia africana (L.) R.Br. & Herb & $\mathrm{N}$ & Th \\
\hline & & 60 & Malcolmia cubulica L. & Herb & Mic & Th \\
\hline & & 61 & Nasturtium officinale $\mathrm{R} . \mathrm{Br}$ & Herb & L & $\mathrm{Np}$ \\
\hline & & 62 & Raphanus sativa L. & Herb & L & Th \\
\hline 16 & $\begin{array}{l}\text { Canabac } \\
\text { eae }\end{array}$ & 63 & Canabis sativa L. & Shrub & Mic & Th \\
\hline 17 & $\begin{array}{l}\text { Cactacea } \\
\text { e }\end{array}$ & 64 & Opuntia dillenii L. & Shrub & Mes & Th \\
\hline \multirow[t]{6}{*}{18} & \multirow{6}{*}{$\begin{array}{l}\text { Chenopo } \\
\text { diaceae }\end{array}$} & 65 & Chenopodium ambrisiodes L. & Herb & L & Th \\
\hline & & 66 & Chenopodium album L. & Herb & Mic & Th \\
\hline & & 67 & Chenopodium foliosum L. & Herb & $\mathrm{N}$ & Th \\
\hline & & 68 & Chenopodium murale L. & Herb & L & Th \\
\hline & & 69 & Salsola imbricata Forssk. & Herb & $\mathrm{L}$ & Th \\
\hline & & 70 & Sueada fruiticosa L. & Shrub & $\mathrm{N}$ & Th \\
\hline \multirow[t]{3}{*}{19} & \multirow{3}{*}{$\begin{array}{l}\text { Caryoph } \\
\text { yllaceae }\end{array}$} & 71 & Spergula arvensis L. & Herb & Mic & Th \\
\hline & & 72 & Silene conoidea L. & Herb & Mic & Th \\
\hline & & 73 & Stellaria media (L.) Vill. & Herb & $\mathrm{L}$ & $\mathrm{H}$ \\
\hline 20 & $\begin{array}{l}\text { Combret } \\
\text { aceae }\end{array}$ & 74 & Conocarpus lancifolius Engl. & Tree & $\mathrm{N}$ & G \\
\hline \multirow[t]{3}{*}{21} & \multirow{3}{*}{$\begin{array}{l}\text { Convolv } \\
\text { ulaceae }\end{array}$} & 75 & Convolvulus arvensis $\mathrm{L}$. & Herb & L & Th \\
\hline & & 76 & Convolvulus prostrates Forssk & Herb & L & Th \\
\hline & & 77 & Ipomoea carnea Jace. & Herb & L & Th \\
\hline \multirow[t]{4}{*}{22} & \multirow[t]{4}{*}{$\begin{array}{l}\text { Cucurbit } \\
\text { aceae }\end{array}$} & 78 & $\begin{array}{l}\text { Citrullus colocynthis (L.) } \\
\text { Schard }\end{array}$ & Herb & Mic & Th \\
\hline & & 79 & Cocurbita pepo L. & Herb & $\mathrm{Mg}$ & Th \\
\hline & & 80 & Luffa cylendrica L. & Herb & $\mathrm{Mg}$ & Th \\
\hline & & 81 & Silene arenosa C.Koch & Herb & $\mathrm{N}$ & Th \\
\hline 23 & Euphorbi & 82 & Euphorbia helioscopia L. & Herb & $\mathrm{L}$ & Th \\
\hline
\end{tabular}


272 Fawad Khan, Zahir Muhammad et. al. Florisitic Composition and Ecological ...

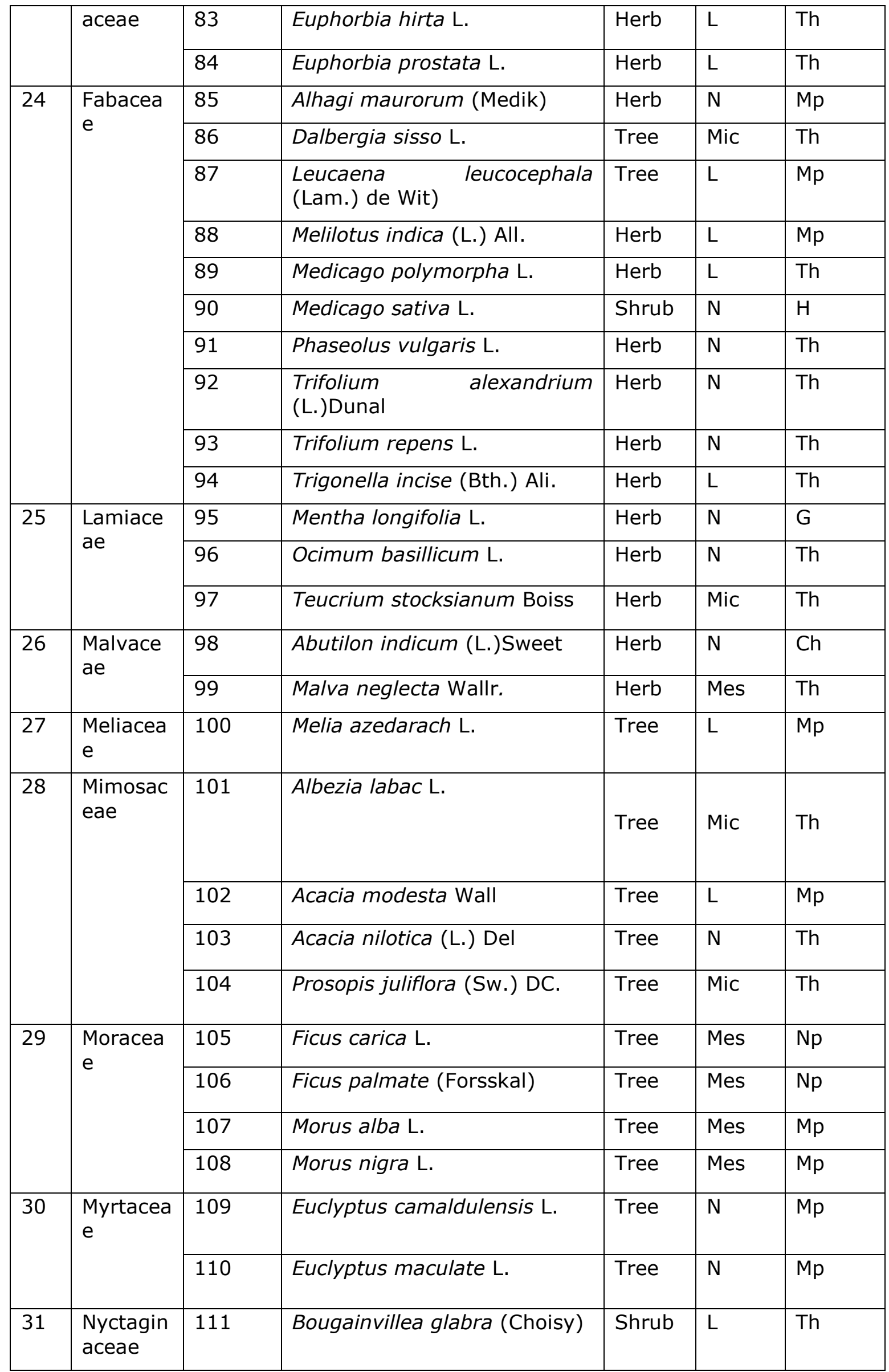




\begin{tabular}{|c|c|c|c|c|c|c|}
\hline \multirow[t]{2}{*}{32} & \multirow[t]{2}{*}{ Oleaceae } & 112 & Jasminium mesnii L. & Shrub & Mic & $\mathrm{Mp}$ \\
\hline & & 113 & Jasminum sambac (L.) Aiton & Shrub & Mic & $\mathrm{Mp}$ \\
\hline 33 & $\begin{array}{l}\text { Papaver } \\
\text { aceae }\end{array}$ & 114 & Hypocum pendulum L. & Herb & L & Th \\
\hline \multirow[t]{4}{*}{34} & \multirow{4}{*}{$\begin{array}{l}\text { Plantagi } \\
\text { naceae }\end{array}$} & 115 & Bacopa monnieri L. & Herb & Mic & Th \\
\hline & & 116 & Veronica arvensis L. & Herb & Mic & Th \\
\hline & & 117 & Veronica biloba L. & Herb & Mic & Th \\
\hline & & 118 & Plantago lanceolata L. & Herb & Mes & $\mathrm{Mp}$ \\
\hline 35 & $\begin{array}{l}\text { Polygona } \\
\text { ceae }\end{array}$ & 119 & $\begin{array}{ll}\text { Persicaria } & \text { glabra(Willd.) } \\
\text { M.Gómez } & \end{array}$ & Herb & Mic & Th \\
\hline 36 & $\begin{array}{l}\text { Primulac } \\
\text { eae }\end{array}$ & 120 & Anagallis arvensis L. & Herb & $\mathrm{N}$ & Th \\
\hline 37 & $\begin{array}{l}\text { Punicace } \\
\text { ae }\end{array}$ & 121 & Punica granatum L. & Tree & Mic & $\mathrm{Mp}$ \\
\hline \multirow[t]{2}{*}{38} & \multirow{2}{*}{$\begin{array}{l}\text { Rhamna } \\
\text { ceae }\end{array}$} & 122 & Ziziphus mauritiana (Lam). & Tree & $\mathrm{N}$ & $\mathrm{Mp}$ \\
\hline & & 123 & Ziziphus nummlaria L. & Shrub & $\mathrm{N}$ & MP \\
\hline \multirow[t]{3}{*}{39} & \multirow{3}{*}{$\begin{array}{l}\text { Rosacea } \\
\text { e }\end{array}$} & 124 & Pyrus communis L. & Tree & $L$ & Th \\
\hline & & 125 & Rosa alba L. & Shrub & $\mathrm{N}$ & Th \\
\hline & & 126 & Rosa indica L. & Shrub & $\mathrm{N}$ & Th \\
\hline 40 & $\begin{array}{l}\text { Rubiacea } \\
\text { e }\end{array}$ & 127 & Galium aparine L. & Herb & $\mathrm{L}$ & Th \\
\hline 41 & $\begin{array}{l}\text { Ranuncu } \\
\text { laceae }\end{array}$ & 128 & Rannunculus muricatus L. & Herb & Mic & $\mathrm{G}$ \\
\hline 42 & $\begin{array}{l}\text { Salvador } \\
\text { aceae }\end{array}$ & 129 & Salvadora oleoides Decne. & Shrub & Mic & $\mathrm{Mg}$ \\
\hline \multirow[t]{8}{*}{43} & \multirow{8}{*}{$\begin{array}{l}\text { Solanace } \\
\text { ae }\end{array}$} & 130 & Datura metel L. & Herb & Mes & $\mathrm{Ch}$ \\
\hline & & 131 & Lycopersicum esculentum L. & Herb & Mic & Th \\
\hline & & 132 & Physalis divaricata D. Don & Herb & $\mathrm{N}$ & Th \\
\hline & & 133 & Solanum melongena L. & Herb & Mic & $\mathrm{H}$ \\
\hline & & 134 & Solanum nigrum L. & Herb & Mic & $\mathrm{Ch}$ \\
\hline & & 135 & Solanum suratense L. & Herb & $\mathrm{N}$ & Th \\
\hline & & 136 & $\begin{array}{l}\text { Withania coagulans (Stocks) } \\
\text { Dunal }\end{array}$ & Shrub & Mic & $\mathrm{Ch}$ \\
\hline & & 137 & $\begin{array}{l}\text { Withania sominifera } \\
\text { Dunal }\end{array}$ & Shrub & Mic & $\mathrm{Ch}$ \\
\hline 44 & $\begin{array}{l}\text { Spindace } \\
\text { ae }\end{array}$ & 138 & Dodonea viscosa (L.) Jacq. & Shrub & $\mathrm{N}$ & $\mathrm{Np}$ \\
\hline 45 & Verbena & 139 & Lantana camara L. & Shrub & Mic & $\mathrm{Mp}$ \\
\hline
\end{tabular}




\begin{tabular}{|l|l|l|l|l|l|l|}
\hline & ceae & 140 & Phyla nodiflora (L.) Greene & Herb & Mic & Ch \\
\hline 46 & Vitaceae & 141 & Vitis vinifera L. & Tree & N & Th \\
\hline 47 & $\begin{array}{l}\text { Zygophy } \\
\text { Ilaceae }\end{array}$ & 142 & Fagonia indica Hadidi & Herb & L & Th \\
\cline { 3 - 8 } & & 143 & Pegnum harmala L. & Herb & L & H \\
\hline
\end{tabular}

Key:

Life form: $\quad \mathrm{Th}=$ Therophytes, $\mathrm{G}=$ Geophytes, $\mathrm{Ch}=$ Chamaephytes,

$\mathrm{H}=$ Hemicryptophytes, $\mathrm{N}=$ Nanophanerophytes, $\mathrm{Mp}=$

Microphanerophytes, $\mathrm{Mg}=$ Megaphanerophytes

Leaf size: $\quad \mathrm{N}=$ Nanophyll, $\mathrm{Mic}=$ Microphyll, $\mathrm{L}=$ Leptophyll, Mes $=$ Mesophyll

Mac $=$ Macrophyll, Mg= Megaphyll, Ap= Aphyllous

\section{Literature Cited}

Ahmad, S., M Zafar, Ahmad, M. Sultana, S. Yaseen, G. Khan, K. and F. Khan (2021). Health benefits of honey and ethno-botanical uses of its bee flora from Lakki Marwat district, Khyber Pakhtunkhwa, Pakistan. IJABS, 1(7), 27-35.

Ahmad, S., M. Zafar, M. Ahmad, F. A. Ozdemir, G. Yaseen, S. Sultana, and M. A.

Ahmad, W., R. Qureshi, and M. Arshad. 2019. Floristic, Frequency and Vegetatio-Biological Spectra Of

.Ali, S. I. and M. Qaisar. 1995. Flora of Pakistan. Pakistan Agricultural Research Council, Islamabad.

Ali, S.I. and M. Qaiser (Eds.). 19952015. Flora of Pakistan. Department of Botany, University of Karachi. Ali, S.I. and Y.J. Nasir (Eds.). 19891992. Flora of Pakistan Islamabad, Karachi.

Badshah, L., F. Hussain, and Z. Sher. 2013. Floristic inventory, ecological characteristics and biological spectrum of rangeland, District Tank, Pakistan. Pak. J. Bot., 45(4): 1159-1168.

Batalha, M.A. and F.R. Martins. 2002. Life -form spectra of Brazilian cerrado sites. Flora, Morphology, Distribution, Functional Ecology of Plants, 197(6): 452-460.

Batalha, M.A. and F.R. Martins. 2004. Floristic frequency, and vegetation
Kutlu. Palynological studies of winter weeds melliferous flora of district Bannu, Khyber Pakhtunkhwa, Pakistan. Annali di Bot. (2020): 77-86.

Ahmad, S., M. Zafar, M. Ahmad, G. Yaseen, and S. Sultana (2019). Microscopic investigation of palyno-morphological features of melliferous flora of Lakki Marwat district, Khyber Pakhtunkhwa, Pakistan. Micros. Res. Tech. 82(6), 720-730.

Murree kotli Sattian-Kahuta National Park, Pakistan. Pak. J. Bot., 51 (2): 637-648

life form spectra of Cerrado site. Braz. J. Bio., 4(2): 203-209.

Cain, S.A. and G.M. Castro. 1959. Manual of Vegetation analysis. Harper, NYpp. 325.

Durrani, J. M., F. Hussain and S. Rehman. 2005. Ecological Characteristics of Plants of Harboi Rangeland, Kalat, Pakistan. J Trop Subtrop Bot. 13 (2): 130-138.

Fazal, H., N. Ahmad, A. Rashid and S. Farooq. 2010. A checklist of phanerogamic flora of Haripur Hazara, Khyber Pakhtunkhwa, Pakistan. Pak. J. Bot., 42(3): 15111522.

Husain, F., 1989. Field and Laboratory Manual of Plant Ecology. UGC. Islamabad.

Hussain, F., S. M. Shah, F. Hadi, and A. Ullah. 2009. Diversity and ecological 
Characteristics of Wheat Fields of University of Peshawar Botanical Garden at Azakhel, District Nowshera, Pakistan. Pak. J. Weed. Sci. Res., 15 (4): 283-294.

Hussain, T. and M. I. Chaudhry. 2009. A Floristic description of flora and Ethnobotany of Samahni Valley (A.J.K.), Pakistan. Ethnobot. Leaflets . 13: 873-899.

Khan, A., N. Khan, K. Ali and I, Rahman. 2017. Floristic Diversity, Life-Forms and Biological Spectrum of Vegetation in Swat Ranizai, District Malakand, KP, Pakistan. Sci Technol Dev 36 (2): 61-78

Khan, M., F. Hussain and S. Musharaf. 2013. Floristic composition and Biological Characteristics of the vegetation of Sheikh Maltoon town District Mardan, Pakistan. Annual Review And |Research in Biology, 3 (1): 31-41.

Khan, M., F. Hussain and S. Musharaf. 2014. Floristic Composition and Ecological Characteristics of ShahbazGarhi. District Mardan, Pakistan. Global J. of Sci. Frontier research, 14 (1): 7-17.

Marwat, S., K. Khan, M. Ahmad M, Zafar M and K. Usman (2012b). Floristic account of the Asclepiadaceous Species from the Flora of Dera Ismail Khan District, KPK, Pakistan. Am. J. Plant Sci.. 3: 141-149.

Marwat, Q. and R.A. Qureshi. 2000. A check list of the vascular plants found in upper siran reserved and guzara forests, District Mansehra,

Shah, S. M., A. Ullah, and F. Hadi. 2014. Ecologocal characteristics of plants Flora in the Wheat Crop of Mastuj Valley, District Chitral, Khyber Pakhtunkhwa, Pakistan. Pak. J. Weed Sci. Res., 20 (4): 479-487.

Sher, Z., F. Hussian, L. Badshah, and M. Wahab. 2011. Floristic composition, Communities and Ecological characteristics of plants of wheat fields of Lahor, District, Swabi,
Pakistan. Pak. J. Pl. Sci., 6(1-2): 4357.

Musharaf, K,. F. Husain. S. Musharaf and Imdad Ullah. 2011. Floristic composition, life form, and leaf size spectra of the coal mine area vegetation of Dara Adam Khel, Khyber Pakhtonkhwa, Pakistan. J. Biod. Env. Sci, 1(3): 2222-3045.

Musharaf, K., F. Husain and $S$. Musharaf. 2014. Floristic composition and ecological characteristic of Shahbaz Ghari, District Mardan, Pakistan. Glob. J. Sci. Front. Res., 14(1): ISSN: 22494626.

Nasir, E., \& Ali, S. I. (1971). Flora of West Pakistan Department of Botany. University of Karachi, Karachi, 2007, 112-115.

Nasir, Z. A., and S. Sultan. 2002. Floristic composition, Biological and Leaf size spectea of Weeds In Gram, Lentil Mustard and Wheat fields of district Chakwal, Pakistan, Pak. J. Biol. Sci, 5 (7): 758-762.

Parveen, A., G. R. Sarwar and I . Husain. 2008. Plant biodiversity and phytosociological of Dureji (Khirthar Range). Pak. J. Bot., 40(1): 17-24.

Raunkiaer, C., 1934. The life-forms of plants and statistical plant geography being the collected papers of C. Raunkiaer. Clarend on Press, Oxford.

Samad, M., L. Badshah and S. M. Khan. 2018. Biological Spectra of LalaKalay Area Distrist Peshawar Khyber Pakhtunkhwa Province Pakistan.Pak. J. Weed Sci. Res., 24(4): 353-362.

Pakistan. Pak. J. Bot. 43 (6): 28172820.

Manhas, R. K., L. Singh, H. B. Vashista and M. Negi. 2010. Floristic diversity of protected ecosystem of kandi Region of Punjab, India. NY Sci J., pp: 96-103.

Wariss, H. M., S. A. Pirzada, K. Alam, S. Anjum and R. Qureshi. 2014. Flora of Lal Suhanra National Park, Bahawalpur, Punjab, Pakistan. Pak. J. Bot., 46 (4): 1331-1341. 
276 Fawad Khan, Zahir Muhammad et. al. Florisitic Composition and Ecological ...

Qureshi, R., H. Shaheen, M. Ilyas, W. Ahmed and M. Munir. 2014. Phytodiversity and plant life of Khanpur Dam, Khyber Pakhtunkhwa, Pakistan. Pak. J. Bot., 46 (3): 841849.

Shuaib, M., I. Khan, Sharifullah, R. Khan, Hashmat Ullah, S. Mubarik and R. Naz. 2014. Ethnobotanical study of spring flora of Distrct Dir (Lower), Khyber Pakhtunkhwa, Pakistan. Pak. J. Weed Sci. Res., 20 (1): 37-49. 